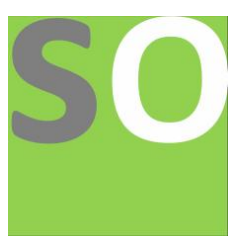

Article title: An experimental conception for proving the Majorana quantum properties of neutrinos Authors: HUANG HANCHENG[1], GUO LIHAN[2], LI MENGXUAN[3], HU XIAOYU[4]

Affiliations: Chongqing DEPU Foreign Language School[1], Chongqing No.1 Secondary School[2]

Orcid ids: 0000-0003-4931-1275[1]

Contact e-mail: 1837503217@qq.com

License information: This work has been published open access under Creative Commons Attribution License http://creativecommons.org/licenses/by/4.0/, which permits unrestricted use, distribution, and reproduction in any medium, provided the original work is properly cited. Conditions, terms of use and publishing policy can be found at https://www.scienceopen.com/.

Preprint statement: This article is a preprint and has not been peer-reviewed, under consideration and submitted to ScienceOpen Preprints for open peer review.

DOI: 10.14293/S2199-1006.1.SOR-.PPEO6IT.v1

Preprint first posted online: 19 February 2020

Keywords: neutrino, Majarana quantum characteristic 


\section{An experimental conception for proving the Majorana quantum properties of neutrinos}

Huang Hancheng ${ }^{1}$ * , Guo Lihsn ${ }^{1}$, Li Mengxuan ${ }^{1}$ and Hu Xiaoyu ${ }^{2}$

1 Chongqing DEPU Foreign Language School, Chongqing, China

2 Chongqing No.1 Secondary School, Chongqing, China

Corresponding author's e-mail address: 1837503217@qq.com

\section{Introduction}

In the late 1930s, Ettore Majorana, a theoretical physicist born in Sicily, Italy, studied quantum particles and suggested the existence of Majorana fermions. Majorana himself disappeared in the following years, but his speculation of Majorana fermions has still had a huge impact on the physics world and made both Majorana fermions and his theory a big hangover in the world[1].

In the early 21 st century, after numerous precise experiments, a 'Majorana-like' fermion was observed in condensation matter materials. At around the same time this, some scholars claimed a new conjecture questioning whether neutrinos are actually Majorana fermions.

Physicists have a long standing argument about this subject, and this conjecture has a significant impact on physics as a whole. For example, if neutrinos are Majorana fermions, neutrinoless double beta decay will then prove that leptons are not conserved[2].

Based on the existing literature and measurement methods, this paper introduces an alternative detection method for identifying potential neutrino quantum characteristics, hoping that some breakthroughs can be made in the detection of neutrinos.

\section{Main problems for experimental testing}

Due to the widespread irregularities that occur in space, background radiation can lead to deviations in experimental results. A way to overcome this difficulty is setting experimental sites to be at a given depth below the ground, with sufficient thickness of soil and rocks to prevent cosmic rays from affecting the accuracy of the 
results. There are quite a few high-energy cosmic rays on the ground, about 200 per square meter per second. These cosmic rays, and the secondary particles and stable isotopes they produce, drown out the rare neutrino signals. Therefore, experiments must be conducted underground, relying on mountains to shield cosmic rays. Some laboratory halls in China are located underground and connected by horizontal tunnels. The rocks on those laboratories are about $150 \mathrm{~m}$ thick[3].

Then actively or passively intercept radiation releases in surface materials, such as surface water and air[4].

When the background radiation from the far end is shielded, the experimental equipment itself may also become a source of radiation. To counter this, the experimental equipment should be carefully screened and cleaned, and storage sites need to take the necessary precautions should be carefully taken care of so as to avoid any contamination of the experimental equipment[5].

When Dry Decontamination is not exhaustive, we must introduce wet Decontamination ensure no radiation remain on the equipment[6] .

Due to the particularity of radiation, the effect that alpha particles have on this experiment can be eliminated by physical interference. But beta and gamma rays can still have a significant impact on the results of the experiment.

The measurement method of the experiment is based on the kinetic energy contained in different electrons, in which the external beta rays directly affect the distribution of electron kinetic energy. The gamma rays can be absorbed by experimental electrons, so that the latter has higher kinetic energy than the original experimental electrons, thus affecting the authenticity of the experimental results.

Therefore, in the selection of radioactive sources for experimental use, with the radioactive sources that can produce higher kinetic energy, beta particles should be selected; or the intensity of background radiation should be reduced. This makes it easier for the beta particles in the experiment to be identified and received by the 
detector. Raw materials without neutrinoless double beta decay also majorly hinder the experiment. For the selection of raw materials, we need to take into account: The $\mathrm{Q}$ value of raw materials, natural abundance, and the compatibility of reaction and measuring instruments[5]. Considering these three factors and to meet the target requirements, we can only choose the elements with low natural abundance and those that possess a higher exposure of the elements. The effect is that the cost of carrying out double beta decay is quite high, including the enrichment of naturally smallabundant isotopes[7]. It is also difficult to conduct large-dose experiments to avoid the effect of experimental errors on the experiment itself.

\section{New experimental design}

In order to avoid a large number of external factors affecting the experimental results, the site of the new experiment must still be set deep underground along with a large amount of vegetation soil and thick rock to isolate interference caused by cosmic rays.

At the same time, the traditional experimental method of neutrino less double beta decay is replaced by two single-beta decay, which overcomes the experimental difficulty and reduces the experimental cost. Here we introduce a new experimental object of electrons, and irradiate a selector that be able to select some electrons with same velocity. Thus, the equal kinetic electron current is provided steadily, and at the same time, by arranging a strong electric field in the space around the beta decay source, the beta particles are produced by the offset decay, so that it does not affect the experimental results. The two beta sources shall be placed at both ends of the incident electron path, and the emitted neutrinos should collide in the space around the electron path. According to quantum field theory, a given field $\varphi$ can produce a particle $A, \bar{A}$ which could annihilate particle $A$, and its' complex conjugate $\varphi^{*}$ can produce $\bar{A}$ to annihilate $\mathrm{A}$

We presume there are some particles, which their antiparticles are themselves, we have $\varphi=\varphi[8]$, if the neutrino is the Majorana fermion, the collision will release the gamma rays. When matter and antimatter collide, they will annihilate each other, and all the matter involved in the annihilating reaction will be converted into the energy of high-energy $\gamma$ photon, which radiates the material 
world and the antimatter world[9], that have been absorbed by electrons and allowed them to gain higher kinetic energy. The electrons on the electronic path will be measured on the device's final screen.

More details about this experiment as follows:

The high-pressure mercury lamp irradiates the metal in the experiment, releasing the quantitative kinetic energy electrons.

Currently interval, according to Einstein's photoelectric effect equation, we have:

(1) $\boldsymbol{K}_{\boldsymbol{E}}=\boldsymbol{h} \boldsymbol{f}-\boldsymbol{W}$

Where, $\mathrm{W}$ is a size function which is related to the different metals used in experiments, and the function holds as below:

(2) $W=h f_{0}$

Where, $f_{0}$ represents the extreme frequency of the metal in this equation; therefore, equations (1) and (2) can be combined so that the electrons released by different metals have kinetic energy:

$$
\text { (3) } K_{E}=h\left(f-f_{0}\right)
$$

The electron hits the screen alone, which calculates the kinetic energy of the electron.

Two radioactive sources at different locations undergo single-beta decay, and the nuclear decay of the radioactive source releases electrons and neutrinos.

At this point, there is an electronic kinetic energy:

$$
\text { (4) } K_{E e}=E_{B 1}-E_{B 2}-K_{E v}-K_{E n}
$$

Where, $\mathrm{K}_{\mathrm{Ee}}$ represents the electron kinetic energy, $\mathrm{E}_{\mathrm{B} 1}$ indicates the 
decay of the pre-decay atomic nuclear binding energy, $\mathrm{E}_{\mathrm{B} 2}$ indicates the decay of the nuclear binding energy. $\mathrm{K}_{\mathrm{Ev}}$ indicates the decay to produce the kinetic energy of neutrinos, and $\mathrm{K}_{\mathrm{En}}$ indicates the decay of the atomic nuclear kinetic energy. By rearranging the formula we can obtain:

(5) $K_{E v}=E_{B 1}-E_{B 2}-K_{E n}-K_{E e}$

The electrons of the product of nuclear decay will be affected by a strong electric field in the space surrounding the radioactive source, thus keeping the product electron away from the incident orbit of the experimental electron.

At this point, we can get:

(6) $W=K_{E e}=\int E q d x$

In this formula, E represents the strength of the electric field, $\mathrm{q}$ represents the electronic charge, and $\mathrm{W}$ represents the electric field.

According to this formula, you can get:

(7) $\frac{d K_{E e}}{d X}=E q$

(8) $E=\frac{d K_{E e}}{d X q}$

$\mathrm{E}$ is the electric field strength required to produce a strong electric field around the decay source.

On both sides, neutrinos produced by nuclear decay will meet and collide near the incoming electron orbit. At this point, if they are indeed the same as Majorana fermions, gamma rays will be released.

Because neutrinos have so little mass, the frequency of gamma rays produced by their annihilation can be obtained from:

(9) $E=h f=2 m_{v} c^{2}+2 E_{K v}$ 
Because the nucleus and the kinetic energy carried by electrons are easier to measure, the formula can be integrated to obtain:

$$
\text { (10) } E=h f=2 m_{v} c^{2}+2\left(E_{B 1}-E_{B 2}-K_{E n}-K_{E e}\right)
$$

At this point, because neutrinos are too low a mass, we can't directly measure their static mass. But we can estimate the neutrino mass by observing experimental results in neutrino less double-beta decay. Here there can be the equation:

$$
\text { (11) } T_{1 / 2}^{0 v}=G^{0 v}(Q, Z)\left|M^{0 v}\right|^{2}\left(\frac{\langle M \beta \beta\rangle}{m_{2}}\right)^{2}
$$

Then you can get:

$$
\langle M \beta \beta\rangle=\left|\sum_{j} m_{j} U_{e j}^{2}\right|=\left|u_{e 1}^{2} 3^{i a 1} m_{1}+u_{e 2}^{2} 3^{i a 2} m_{2}+u_{e 3}^{2} m_{3}\right|
$$

At the same time:

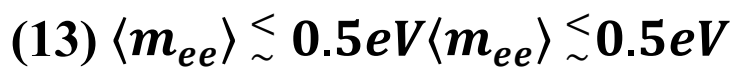

At this point, bring this result into our formula, and in order to ensure the reliability of the data, we can take:

(14) $m_{e e}=0.25 e V$

Then, according to

$$
\text { (15) } E=h f=2 m_{v} c^{2}+2\left(E_{B 2}-K_{B 2}-K_{E n}-K_{E e}\right)
$$

There is:

$$
\text { (16) } E=h f=0.5 e V+2\left(E_{B 1}-K_{B 2}-K_{E n}-K_{E e}\right)
$$

Taking into account possible background radiation and other issues, this energy should be higher than the effect of background radiation on electrons, then:

$$
\text { (17) } 2\left(E_{B 1}-E_{B 2}-K_{E n}-K_{E e}\right)>2 e V
$$


Such an energy standard is not worth mentioning as compared to the total decay energy of beta decay, in the case of ${ }^{120} \mathrm{ba},{ }^{120} \mathrm{ba}$ 's total beta decay energy is about $5 \mathrm{MeV}$. This is far more than the experimental need which is shorter than $2 \mathrm{ev}$. Even if that can be met, neutrinos cannot carry $5 \mathrm{MeV}$ energy.

However, due to the particularity of beta decay, in which neutrinos carry an indeterminate energy, we can solve the problem of neutrino carrying energy through the tonnage experiment.

At the same time, the experimental electrons running in the electron orbit will absorb the gamma rays produced by neutrino annihilation, thereby converting the pure energy contained in the gamma rays into its own kinetic energy, and the screen will accept the electron kinetic energy:

$$
\text { (18) } K_{E e}=h f-W+0.5 e V+2\left(E_{B 1}-K_{B 2}-K_{E n}-K_{E e}\right)
$$

The screen receives different kinetic electrons, thus proving the quantum properties of electrons.
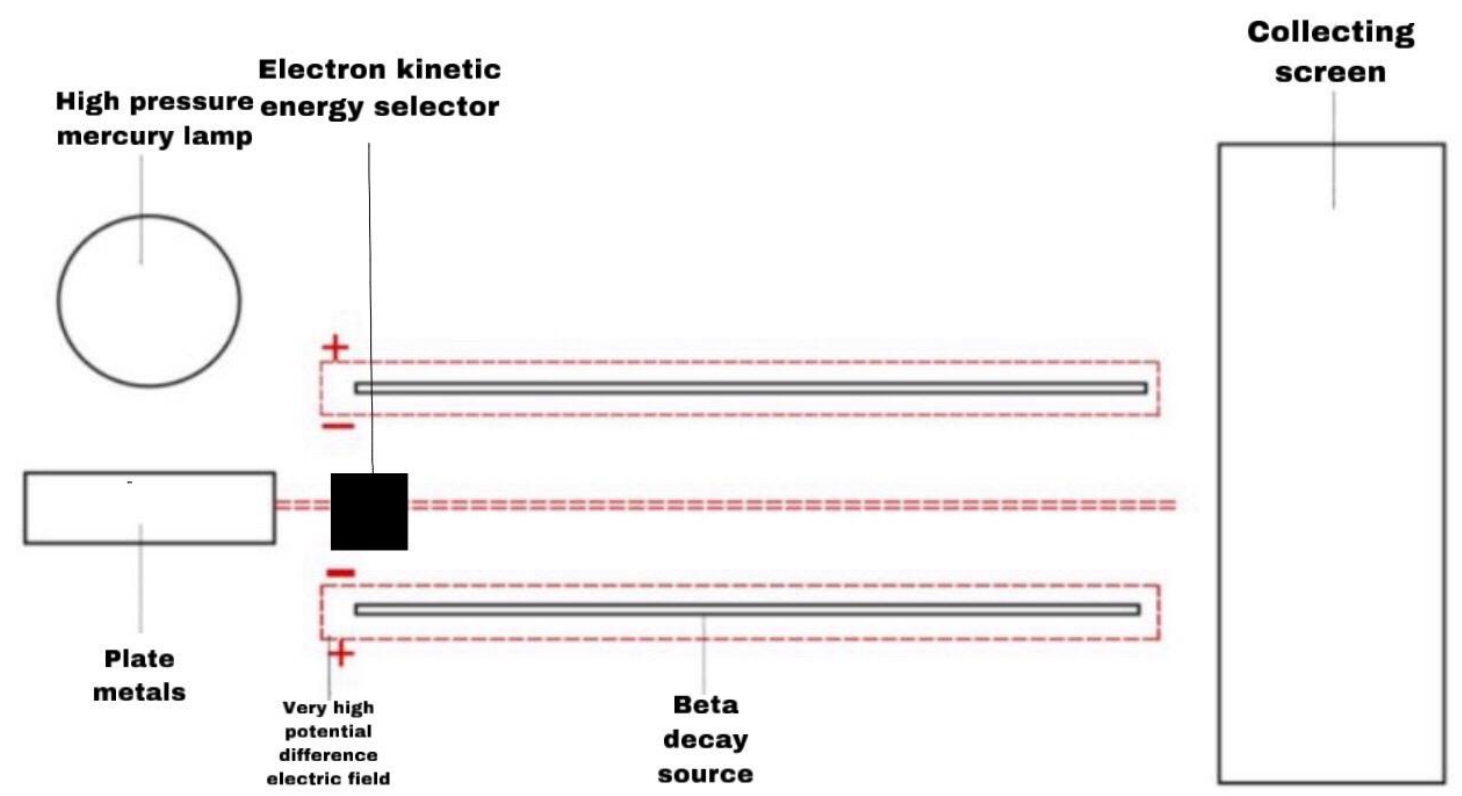

Figure 1 Experimental Overall Style

\section{Ideal experiments and their results}

The following experimental results are based on theoretical derivations, and their experimental models are based on the new 
experimental methods mentioned in figure 2 , for reference only.

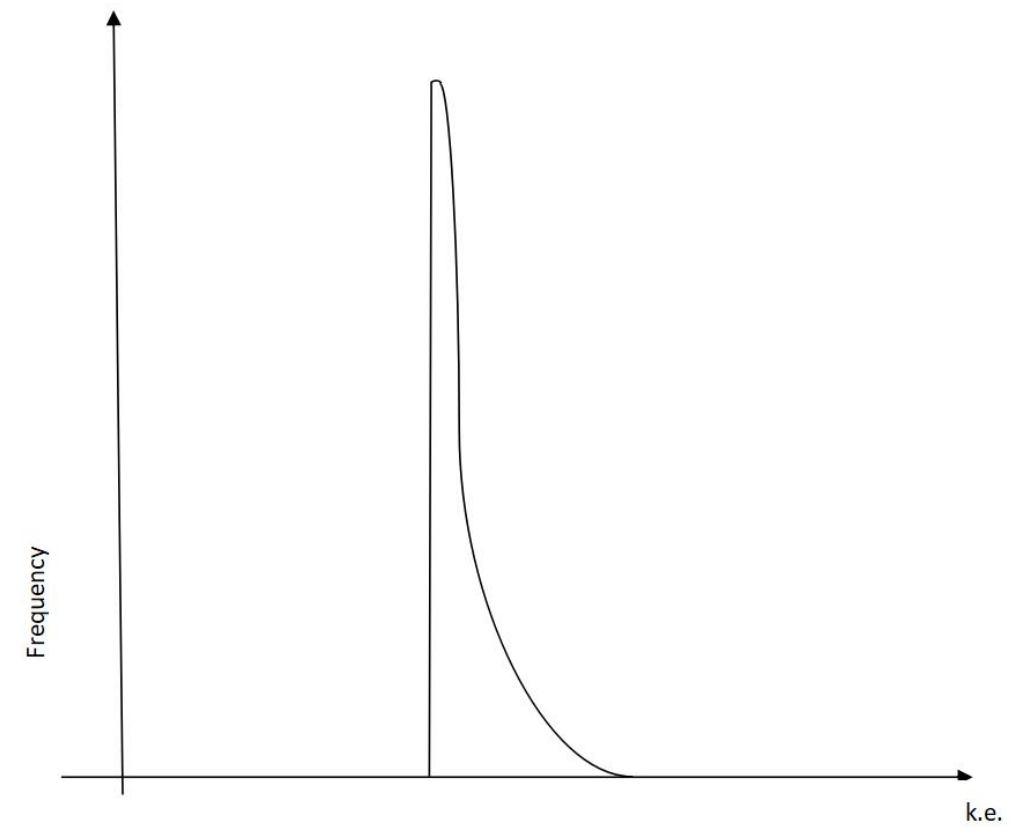

Figure 2 experimental results

The peak of the electronic kinetic energy in the figure is the experimental electron kinetic energy that has not absorbed any beta decay-released gamma rays, which account for the majority of the total electron. This is because of most electrons do not absorb gamma rays in orbit relative to the entire electron, and the lowering function on the right side of the peak is the kinetic energy distribution of the affected electrons.

\section{Conclusion}

Thus far, the most viable experiment has been the neutrino less double beta decay. A large number of neutrinos in the experiment do not move away from the collision path of the ideal experiment according to the ideal orbit, so the control of the path of neutrinos will be an important technical challenge. If more accurate data is obtained, and if the neutrinos are Majorana fermion, the experimental data will be very similar to the ideal results. Such an outcome would have a huge impact and revelation in the modern physics community. People will learn more about the elementary particles, and the characteristics of the Majorana fermion, which will allow humans to break the law of lepton.

\section{References:}

[1] Liu Jianglai. No neutrino double beta decay. Modern physics 
knowledge.2016 Vol.27 (6): 52-56

[2] Weeks Shuhua. Look for neutrino-free double beta decay. Physical. 2018 Vol. 47 (5): 325-325

[3] Cao J. Daya Bay and Jiangmen Underground Neutrino Observat ory(JUNO) neutrino experiments . Sci SinPhys Mech Astron, 2014, 44: 10251040, doi: 10.1360/SSPMA 2014-00174

[4] Andrea Giuliani and Alfredo Poves. Neutrinoless Double-Beta Decay. Hindawi PublicIng Corporation in High Energy Physics Volume 2012

[5] Laura Cardani. Neutrinoless double beta decay overview. SciPost Phys. Proc. 1, 024 (2019)

[6] https://kns.cnki.net/KCMS/detail/detail.aspx?Dbcode $=$ CJFQ\& dbname $=$ CJFDLAST2019\&filename $=$ FGGL201929039 $\& \mathrm{~V}=\mathrm{M}$ Dk3NDIxTHV4WVM3RGgxVDNxVHJXTTFGckNVUjdxZlp 1WnFGeWpnVzd2QU15ck1Zckc0SDlqT3BvOUdiWVI4ZVg=

[7] Andrea Giuliani and Alfredo Poves. Neutrinoless Double-Beta Decay. Hindawi PublicIng Corporation in High Energy Physics Volume 2012

[8] He Qinglin. Topological superconductivity and Majorana fermion. Chinese Science Bulletin, 2018, 63: 2717-2730

He Q L. Topological superconductivity and Majorana fermion ( in Chinese). Chin Sci Bull, 2018, 63: 27172730, doi: 10.1360/N972018-00555

[9] Yang JiefuO572.11 [A] 1000-2537(1999)04-36-08 